\title{
The $\mathrm{k}: \mathrm{k}+4$ resonances in planetary systems
}

\author{
Benoît Noyelles and Alain Vienne \\ IMCCE - Laboratoire d'Astronomie de Lille, UMR 8028 du CNRS, 1 impasse de \\ l'Observatoire, 59000 Lille, France \\ e-mail : Benoit.Noyelles@imcce.fr
}

\begin{abstract}
In this study we give a first description of De Haerdtl's 3:7 inequality between the Jovian satellites Ganymede and Callisto and 1:5 inequality between the Saturnian Titan and Iapetus and the resonant arguments associated. For each inequality, 19 arguments are associated. The overlapping of resonant zones induces stochasic layers that the system might have crossed in the past thanks to tidal dissipation.
\end{abstract}

Keywords. Celestial mechanics, planets and satellites: individual: Jupiter - Saturn

\section{Introduction}

The problem of high order resonances has often been studied in the case of resonances between asteroides and Jupiter (see for instance Moons \& Morbidelli (1995)) but there might have been such resonances in satellite systems, since there is a 3:7 great inequality (De Haerdtl's inequality, see De Haerdtl (1892)) between the Jovian satellites Ganymede and Callisto and 1:5 inequality between the Saturnian ones Titan and Iapetus. The case of satellites is very different from asteroides because there are not 3-bodies restricted problems, so there are much more arguments involved, but there are fewer bodies. This study is based on Ganymede - Callisto and Titan - Iapetus problems.

\section{Physical description of the two systems}

Physical and dynamical data related to Ganymede-Callisto and Titan-Iapetus are listed in Tables 1 and 2. It is important to notice that the ratio of the mean motions of Ganymede and Callisto is very near from $\frac{3}{7}$ and the one of Titan and Iapetus very near from $\frac{1}{5}$. The mass ratios are important too. The one of Ganymede-Callisto is near 1, so this problem is very different from an asteroide-Jupiter problem, which can be modeled by a 3-bodies restricted problem. The one of Titan-Iapetus is here 76 but could be evaluated between 45 and 125 because of a large uncertainty on Iapetus' mass.

Table 1. Physical data related to Ganymede and Callisto. The mean motions, semimajor axis and inclinations come from E5 ephemerides (Lieske (1998)), the eccentricities from Burns (1986), the mean radii from the IAU recommandations (Seidelmann et al. (2002)) and the masses from Pioneer and Voyager spacecrafts (Campbell \& Synnott (1985)).

\begin{tabular}{l|cc}
\hline & Ganymede & Callisto \\
\hline Mean motion n $\left(\mathrm{rad} . \mathrm{d}^{-1}\right)$ & 0.878132432119 & 0.376443069266 \\
Semi-major axis a $(\mathrm{km})$ & 1070427.5 & 1882758.6 \\
Eccentricity e & 0.0021 & 0.007 \\
Inclination i & $11^{\prime} 8^{\prime \prime}$ & $15^{\prime} 10^{\prime \prime}$ \\
Mean radius r $(\mathrm{km})$ & 2632.345 & 2409.3 \\
Mass $\mathrm{m}\left(M_{j}\right)$ & $7.80431 \times 10^{-5}$ & $5.66832 \times 10^{-5}$ \\
\hline
\end{tabular}


Table 2. Physical data related to Titan and Iapetus. The mean motions, semi-major axis, eccentricities, inclinations as well as Iapetus' mass come from TASS1.6 (Vienne \& Duriez (1995)), whereas Titan's mass comes from Pioneer and Voyager data (Campbell \& Anderson (1989)) and the mean radii from the IAU recommamdations (Seidelmann et al. (2002)).

\begin{tabular}{l|cc}
\hline & Titan & Iapetus \\
\hline Mean motion n $\left(\mathrm{rad} . \mathrm{d}^{-1}\right)$ & 0.3940956849 & 0.07924104005 \\
Semi-major axis a $(\mathrm{km})$ & 1221728.7 & 3559387.7 \\
Eccentricity e & 0.0289 & 0.029 \\
Inclination i & $27^{\prime}-50^{\prime}$ & $11^{\circ}-18^{\circ}$ \\
Mean radius r $(\mathrm{km})$ & 2575 & 718 \\
Mass m $\left(M_{s}\right)$ & $2.36638 \times 10^{-4}$ & $3.1 \times 10^{-6}$ \\
\hline
\end{tabular}

\section{Location of the resonances}

The common point between two groups of resonances of the same order (here 4) is that they have the same number of arguments, every argument being likely to generate a resonance if considered isolated. For $\mathrm{k}: \mathrm{k}+4$ resonances, there are 19 arguments of the form:

$$
\phi=k \lambda-(k+4) \lambda^{\prime}+q_{1} \varpi+q_{2} \varpi^{\prime}+q_{3} \Omega+q_{4} \Omega^{\prime}
$$

where $q_{1}+q_{2}+q_{3}+q_{4}=4$ and $q_{3}+q_{4}$ is even, so as to respect D'Alembert law. At the middle of the resonance zone, $\langle\dot{\phi}\rangle=0$, what gives:

$$
k n-(k+4) n^{\prime}+q_{1} \dot{\varpi}+q_{2} \dot{\varpi}^{\prime}+q_{3} \dot{\Omega}+q_{4} \dot{\Omega}^{\prime}=0
$$

A knowledge of the velocities of nodes and pericentres could allow us to determine a ratio $\alpha$ between the semi-major axis, deduced from the mean motions. Velocities of the nodes and pericentres (Table 3) come from Lainey's ephemerides (Lainey, Arlot \& Vienne (2004)) for Ganymede and Callisto and TASS1.6 (Vienne \& Duriez (1995)) for Titan and Iapetus.

Table 3. Estimations of velocities of pericentres and nodes of the involved satellites in the laplacian plane

\begin{tabular}{l|cc}
\hline & Pericentre & Node \\
\hline Ganymede & $1.27274 \times 10^{-4} d^{-1}$ & $-1.24913 \times 10^{-4} d^{-1}$ \\
Callisto & $3.20651 \times 10^{-5} d^{-1}$ & $-3.05609 \times 10^{-5} d^{-1}$ \\
Titan & $2.44596 \times 10^{-5} d^{-1}$ & $-2.44524 \times 10^{-5} d^{-1}$ \\
Iapetus & $5.40641 \times 10^{-6} d^{-1}$ & $-5.27184 \times 10^{-6} d^{-1}$ \\
\hline
\end{tabular}

So as to really model the location of resonances, we have to evaluate their width thanks to Eq. (3.3) from Champenois \& Vienne (1999) giving the $\alpha$ 's amplitude for an isolated resonance:

$$
\Delta \alpha=4 k^{\frac{2}{3}}(k+4)^{-\frac{5}{3}} y \sqrt{\frac{1}{3}\left[k^{2} \alpha_{r}^{-2} m^{\prime}+(k+4)^{2} m\right]\left|f\left(\alpha_{r}\right)\right|}
$$

where

$$
y=\sqrt{e^{\left|q_{1}\right|} e^{\prime\left|q_{2}\right|} \gamma^{\left|q_{3}\right|} \gamma^{\prime}\left|q_{4}\right|} \text { with } \gamma=\sin \frac{i}{2}
$$




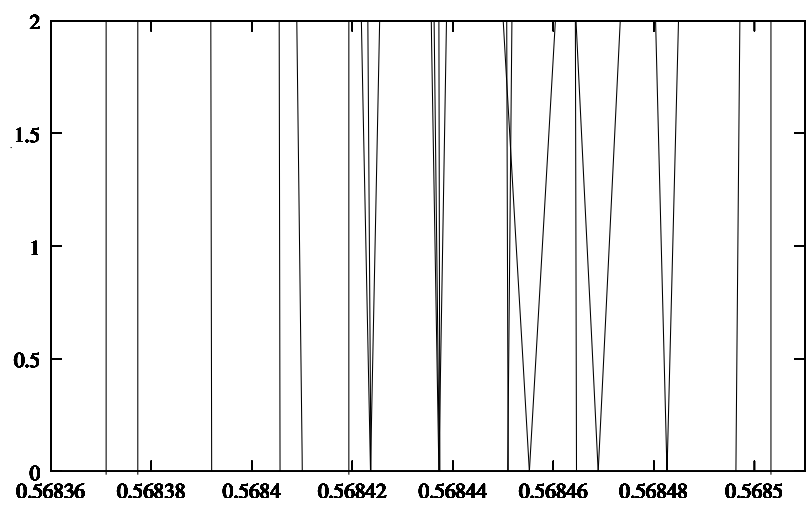

Figure 1. Locations of the resonance for the case Ganymede - Callisto. The $\mathrm{x}$-axis is $\alpha$, the $\mathrm{y}$-axis is $\frac{y}{y_{1}}$ where $y_{1}$ is $y$ (see Eq.3.4) evaluated with $e=0.0021, e^{\prime}=0.007, \gamma=0.0016$ and $\gamma^{\prime}=0.0022$ (actual values of these parameters).

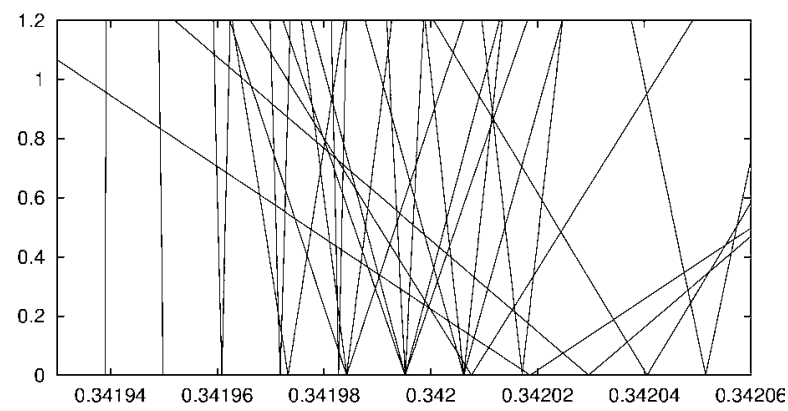

Figure 2. Locations of the resonance for the case Titan-Iapetus. Here $y_{1}$ is evaluated with $e=0.289, e^{\prime}=0.029, \gamma=0.055$ and $\gamma^{\prime}=0.012$. These values correspond to actual values except of Iapetus' inclination which is nowadays 10 times bigger; its actual value maybe due to the crossing of this chaotic zone.

Moreover, $\alpha_{r}$ is $\alpha$ 's value at the exact resonance (say, $\langle\dot{\phi}\rangle=0$, see also Eq. (3.2), and $f$ is a function of $\alpha$ appearing in the developpement of the disturbing function, see for instance Champenois \& Vienne (1999).

When two resonances overlap, as in Figures 1 and 2, a stochastic layer appears (see Chirikov (1979)). In our cases, there are always at least three overlaps due to the quasi equality $\langle\dot{\varpi}+\dot{\Omega}>=0$, but more may appear due to significant values of eccentricities and inclinations that enlarge the resonance zones.

\section{A first exploration of the resonance zones}

\subsection{Migration due to tidal effects}

The two systems are not actually in the resonance zones described above, the actual values of $\alpha$ being respectively 0.56854 for Ganymede-Callisto and 0.34324 for TitanIapetus. Migration of the systems is mostly due to tidal dissipation in the satellites and planets, which provokes change in semi-major axes and so in the mean motions as in Eq. (4.1) taken from Kaula (1964) and Peale \& Cassen (1978):

$$
\frac{\dot{n}}{n}=-\frac{9 k_{2} n m}{2 Q M}\left(\frac{r}{a}\right)^{5}\left[1+\frac{51 e^{2}}{4}\right]+\frac{63 k_{2}^{*} n M}{2 Q^{*} m}\left(\frac{r^{*}}{a}\right)^{5} e^{2}
$$


where ${ }^{*}$ refers to the satellite, $k_{2}$ and $k_{2}^{*}$ are Love numbers, $Q$ and $Q^{*}$ are dissipation functions, and $r$ and $r^{*}$ are the mean radii. This formula works for Callisto, Titan and Iapetus but not for Ganymede since the laplacian resonance with Io and Europa provokes energy transfer to Ganymede (see Yoder (1979)), in fact Ganymede's secular acceleration depends strongly on Io's, as shown by Lainey's numerical simulations (Lainey (2002)). So, we can estimate Ganymede's secular acceleration as $-10^{-11} \cdot y^{-1}$, Callisto's as $\pm 2 \times$ $10^{-14} \cdot y^{-1}$ and Iapetus' as $10^{-19}-10^{-18} \cdot y^{-1}$. The value of Titan's is very important because it determines whether the system has crossed the 1:5 stochastic zone or not. With Eq. (4.1) this quantity can be estimated as $\pm 10^{-12} \cdot y^{-1}$ but only a negative value is compatible with the hypothesis that the system has crossed the stochastic layer, which gives a condition on geophysical parameters of Saturn and Titan, more precisely:

$$
0.0178 \frac{k_{2}^{*}}{Q^{*}}<\frac{k_{2}}{Q}
$$

\subsection{Analytical study of some resonances}

To describe analytically the systems, we used the second fundamental model of the resonance (see Henrard \& Lemaître (1983)). First of all, we expressed the hamiltonian of the system in Jacobi variables while keeping only the terms associated to the studied inequality and the secular terms, which gave :

$$
\begin{aligned}
& H\left(S_{1}, S_{2}, S_{3}, S_{4}, \Phi_{1}, \Phi_{2}, s_{1}, s_{2}, s_{3}, s_{4}, \phi_{1}, \phi_{2}\right)=\Gamma_{0}+\sum_{i=1}^{4} \Gamma_{i} S_{i}+\sum_{i=1}^{4} \Gamma_{i i} S_{i}^{2} \\
& +\sum_{j=i+1}^{4} \sum_{i=1}^{4} \Gamma_{i j} S_{i} S_{j}+K_{4000} S_{1}^{2} \cos \left(4 s_{1}\right)+K_{3100} S_{1}^{\frac{3}{2}} \sqrt{S_{2}} \cos \left(3 s_{1}+s_{2}\right) \\
& +K_{2200} S_{1} S_{2} \cos \left(2 s_{1}+2 s_{2}\right)+K_{1300} \sqrt{S 1} S_{2}^{\frac{3}{2}} \cos \left(s_{1}+3 s_{2}\right)+K_{0400} S_{2}^{2} \cos \left(4 s_{2}\right) \\
& +K_{0040} S_{3}^{2} \cos \left(4 s_{3}\right)+K_{0022} S_{3} S_{4} \cos \left(2 s_{3}+2 s_{4}\right)+K_{0004} S_{4}^{2} \cos \left(4 s_{4}\right) \\
& +K_{1111} \sqrt{S_{1} S_{2} S_{3} S_{4}} \cos \left(s_{1}+s_{2}+s_{3}+s_{4}\right)+K_{2020} S_{1} S_{3} \cos \left(2 s_{1}+2 s_{3}\right) \\
& +K_{2011} S_{1} \sqrt{S_{3} S_{4}} \cos \left(2 s_{1}+s_{3}+s_{4}\right)+K_{2002} S_{1} S_{4} \cos \left(2 s_{1}+2 s_{4}\right) \\
& +K_{0220} S_{2} S_{3} \cos \left(2 s_{2}+2 s_{3}\right)+K_{0211} S_{2} \sqrt{S_{3} S_{4}} \cos \left(2 s_{2}+s_{3}+s_{4}\right) \\
& +K_{0202} S_{2} S_{4} \cos \left(2 s_{2}+2 s_{4}\right)+K_{1120} \sqrt{S_{1} S_{2}} S_{3} \cos \left(s_{1}+s_{2}+2 s_{3}\right) \\
& +K_{1102} \sqrt{S_{1} S_{2}} S_{4} \cos \left(s_{1}+s_{2}+2 s_{4}\right)+K_{0031} S_{3}^{\frac{3}{2}} \sqrt{S_{4}} \cos \left(3 s_{3}+s_{4}\right) \\
& +K_{0013} \sqrt{S_{3}} S_{4}^{\frac{3}{2}} \cos \left(s_{3}+3 s_{4}\right)+\sqrt{S_{1} S_{2}}\left(C_{0}+\sum_{i=1}^{4} C_{i} S_{i}\right) \cos \left(s_{1}-s_{2}\right) \\
& +\sqrt{S_{3} S_{4}}\left(D_{0}+\sum_{i=1}^{4} D_{i} S_{i}\right) \cos \left(s_{3}-s_{4}\right)+E S_{1} S_{2} \cos \left(2 s_{1}-2 s_{2}\right)+F S_{3} S_{4} \cos \left(2 s_{3}-2 s_{4}\right) \\
& +G \sqrt{S_{1} S_{2} S_{3} S_{4}} \cos \left(s_{1}+s_{2}-s_{3}-s_{4}\right)+I \sqrt{S_{1} S_{2} S_{3} S_{4}} \cos \left(s_{1}-s_{2}-s_{3}+s_{4}\right) \\
& +J \sqrt{S_{1} S_{2} S_{3} S_{4}} \cos \left(s_{1}-s_{2}+s_{3}-s_{4}\right)+K S_{1} S_{3} \cos \left(2 s_{1}-2 s_{3}\right) \\
& +L S_{1} S_{4} \cos \left(2 s_{1}-2 s_{4}\right)+M S_{2} S_{3} \cos \left(2 s_{2}-2 s_{3}\right)+N S_{2} S_{4} \cos \left(2 s_{2}-2 s_{4}\right)
\end{aligned}
$$


where $S_{1}$ and $S_{2}$ are proportional to the squares of eccentricities, $S_{3}$ and $S_{4}$ to the squares of inclinations, the 19 first angular terms are related to the great inequality, and the others are secular terms.

Our first study consists in studying a resonance as isolated, so all angular terms disappear except one. The case of arguments like $4 s_{i}$ is the easiest to study and has already been studied by Lemaitre (1984) because it is a reliable modelization of the 3-bodies restricted problem where only one argument is significant.

In such a case, the hamiltonian becomes (second fundamental model of the resonance):

$$
H=\alpha S+\beta S^{2}+4 \epsilon S^{2} \cos 4 s
$$

which becomes

$$
K=R^{2}-\delta R+b R^{2} \cos 4 r
$$

after the canonical transformation

$$
\begin{gathered}
R=S \\
r=(\operatorname{sign} \beta) s \text { if } \beta \epsilon>0 r=(\operatorname{sign} \beta) s-\frac{\pi}{4} \text { if } \beta \epsilon<0
\end{gathered}
$$

with

$$
\begin{aligned}
\delta & =-\frac{\alpha}{\beta} \\
b & =4\left|\frac{\epsilon}{\beta}\right|
\end{aligned}
$$

The exact resonance is reached when $\delta=0$. When $\delta<0$, the argument circulates and when $\delta>0$ the phase space is divided into 3 zones: 2 circulation zones (one prograde and one retrograde) and a resonance zone. Since the resonance zone and the retrograde circulation zone appear at the same time, the capture into resonance is always probabilistic, the probability depending on the evolution of the areas of the critical zones (see Figure 3).

When the hypothesis of the adiabatic invariant is reliable, the probability of capture can be expressed as

$$
P=\frac{2 \pi-2 \arccos \frac{3 b-1}{1+b}}{\pi\left(2-b^{2}\right)-\arccos \frac{3 b-1}{1+b}}
$$

when $\delta$ is increasing. If this expression gives a negative result, $P=0$, and if it gives something bigger than $1, P=1$. When $\delta$ is decreasing, capture is impossible.

In our cases, $P$ can be estimated at a few percents.

\section{Conclusion}

This study shows that 4-order resonances in satellites systems might have played a significant role in the dynamical history of the systems and it is worth to continue the study, while using proper elements instead of nodes and pericentres which are much more representative of the resonant arguments. We also have to check the hypothesis of the adiabatic invariant. If this hypothesis is wrong, probabilities of capture may change (see Gomes (1997)). An exhaustive study of every arguments and their overlaps, as we plan, will precise the role of these inequalities, and maybe will explain Iapetus' actual inclination. 


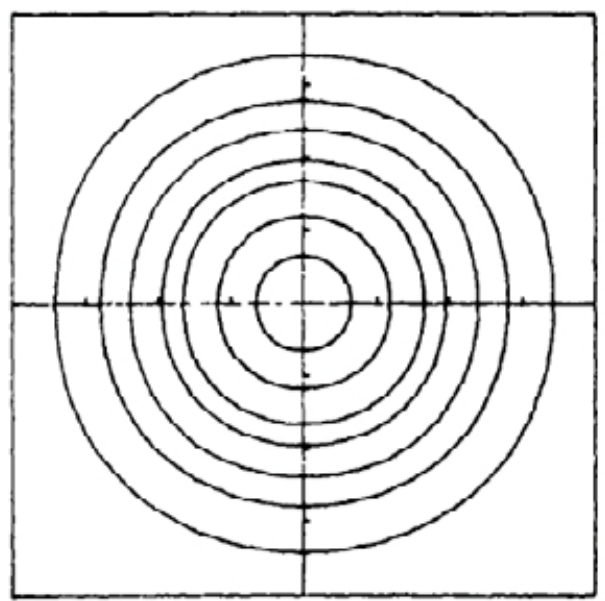

DELTA : -2.0

B : 0.05

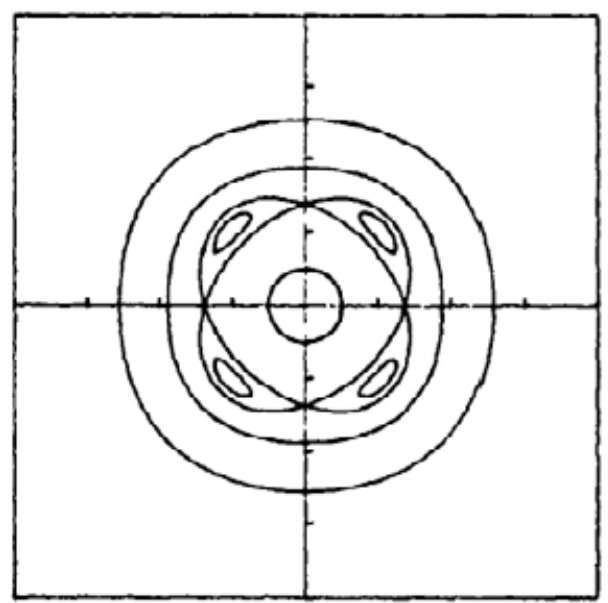

DELTA : 2.0

B : 0.05

Figure 3. The phase space in the case of an isolated resonance of the form $4 \mathrm{~s}$, taken from Lemaitre (1984). On the left there is only one circulation zone, whereas on the right the resonance zone has appeared with its 4 stable equilibria. These figures have been obtained using the Poincaré's variables $x=\sqrt{2 R} \cos r$ and $y=\sqrt{2 R} \sin r$.

\section{References}

Burns, J.A. 1986, in: J.A. Burns \& Matthews, eds. Satellites, Univ. Arizona Press

Campbell, J.K. \& Anderson, J.D. 1989, Astron. J. 97, 1485

Campbell, J.K. \& Synnott, S.P. 1985, Astron. J. 1549, 364

Champenois, S. \& Vienne A. 1999, Cel. Mech. Dyn. Astr. 74, 111

Chirikov, B.V. 1979, Physics Reports 52, 263

De Haerdtl, E. 1892, Bulletin Astronomique 9, 2121

Gomes, R.S. 1997, Astron. Astrophys. 121, 960

Henrard, J. \& Lemaître, A. 1983, Celest. Mech. 30, 197

Kaula, W.M. 1964, Review of Geophysics and Space Physics 2, 467

Lainey, V. 2002, PhD thesis, Observatoire de Paris

Lainey, V., Arlot, J.-E. \& Vienne, A. 2004, Astron. Astrophys., 427, 371

Lemaître, A. 1984, Cel. Mech. Dyn. Astr. 32, 109

Lieske, J.H. 1998 Astron. Astrophys. Suppl., 129, 205

Moons, M. \& Morbidelli, A. 1995, Icarus 114, 33

Peale S.J. \& Cassen P. 1978, Icarus 36, 245

Seidelmann, P.K., Abalakin V.K., Bursa M. et al. 2002, Cel. Mech. Dyn. Astr. 82, 83

Vienne, A. \& Duriez, L. 1995, Astron. Astrophys. 297, 588

Yoder, C.F. 1979, Nature 279, 767 